Published in final edited form as:

Science. 2004 October 8; 306(5694): 247-252.

\title{
Rescue of cardiac defects in Id knockout embryos by injection of embryonic stem cells
}

\author{
Diego Fraidenraich ${ }^{1}$, Elizabeth Stillwell ${ }^{1}$, Elizabeth Romero ${ }^{1}$, David Wilkes ${ }^{2}$, Katia \\ Manova $^{3}$, Craig Basson ${ }^{2}$, and Robert Benezra ${ }^{1}$ \\ 1 Cancer Biology and Genetics Program, \\ 3 Molecular Cytology Core Facility, Memorial Sloan-Kettering Cancer Center, and \\ 2 Molecular Cardiology Laboratory, Greenberg Cardiology Division, Department of Medicine, Weill \\ Medical College of Cornell University, New York, New York, USA.
}

\begin{abstract}
We report that Id knockout mouse embryos display multiple cardiac defects but mid-gestation lethality is rescued by injection of 15 wild type ES cells into mutant blastocysts. Myocardial markers altered in Id mutant cells are restored to normal throughout the chimeric myocardium. Intraperitoneal injection of ES cells into female mice prior to conception also partially rescues the cardiac phenotype with no incorporation of ES cells. IGF1, a long-range secreted factor, in combination with WNT5a, a locally secreted factor likely account for complete reversion of the cardiac phenotype. Thus, ES cells have the potential to reverse congenital defects through Id-dependent local and long-range effects in a mammalian embryo.
\end{abstract}

The Id proteins are dominant negative antagonists of basic helix-loop-helix transcription factors and regulate differentiation in multiple lineages $(l)$. Previous studies have shown that Id1-4 are expressed in embryonic tissues during development in partially overlapping patterns (2) and that Id1 and Id3 are detected at mid-gastrulation in the three germ layers (3). In the developing heart, Id1-3 are detected in the endocardial cushion (EC) mesenchyme from embryonic day (E)10.5 through E16.5 (2), but Id4 is absent (2). Here we show that Id1-3 are also expressed in the epicardium and endocardium, but are absent in the myocardium (Fig.S1AC for Id1, Fig.S1D,E for Id3, not shown for Id2). Id1-3 expression becomes confined to the leaflets of the cardiac valves as the atrio-ventricular (AV) EC tissue myocardializes (not shown). Id 1 and Id 3 expression persists in the cardiac valves/endocardium/endothelium/ epicardium at post-natal day (P)7 (not shown).

\section{Double and triple Id KO embryos display severe cardiac defects and die at midgestation}

Id1, Id 2 or Id $3 \mathrm{KO}$ embryos do not exhibit developmental abnormalities, but ablation of two Id genes in any combination (Id1Id2, Id2Id3 or Id1Id3) leads to embryonic lethality by E13.5 (table SI). Id1/Id3 KO embryos display a collapse of brain vasculature with associated hemorrhage and enhanced expression of p16 and bHLH neural factors in the neighboring neuroepithelium by E12-13 (4). We wanted to identify the cause of embryonic lethality common to all KO embryos. Embryos lacking four or five copies of Id1, Id2 and Id3 displayed multiple cardiac abnormalities at E11.5-E13.5 (Fig.1 A,B,D,E,G,H,J,K,M,N for Id1-/-Id3-/ -, Fig.S2C for Id1-/-Id2-/-, Fig.S2D for Id1-/-Id2+/-Id3-/-and Fig.S2F for Id1+/-Id2+/ - Id3-/-). Embryo size was reduced by $10-30 \%$. KO embryos displayed ventricular septal 
defects (VSDs) associated with impaired ventricular trabeculation and thinning of the compact myocardium. Trabeculae had disorganized sheets of myocytes surrounded by discontinuous endocardial cell lining. Cell proliferation in the myocardial wall was defective (\%BrdU: WT: $38 \pm 4 \%$, Id1-/-Id3-l-:23 $\pm 3 \%$, see Fig 1J,K and 1Linset). Outflow tract (OT) atresia was apparent (Fig.1M,N). OT and atrio-ventricular (AV) ECs displayed low cellularity at E11.5, which resulted in hypoplastic ECs in Id1+/-Id2+/-Id3-/- embryos at E13.5 (Fig.S2E,F). The epicardium appeared normal, and EC apoptosis was unaffected (TUNEL, not shown). The impaired development of the myocardium in Id $\mathrm{KO}$ animals even though Ids are not expressed there suggests Ids might participate in molecular signaling between myocardium and the Id+ epicardium/endocardium/EC. Alternatively, loss of Id expression in early myocardial precursors might lead to myocardial defects.

Id1Id2Id3 KOs have severe cardiac malformation as early as E9.5 (Fig.S2A,B). The overall size of the embryo and heart was reduced by $40-60 \%$. The atrium did not separate from the ventricle, and ventricular trabeculation was rudimentary (Fig.S2A,B). No hemorrhage was observed in Id1Id2Id3 KO brains at E9.5 (not shown). No triple KO embryos survived to E11.5 (Table SI). These observations suggest that a defective heart is the primary cause of midgestation lethality.

\section{Injection of ES cells into Id KO blastocysts corrects cardiac defects and rescues embryonic lethality}

Cardiac defects and embryonic lethality was reversed by injecting 15 LacZ-marked ES (R26) cells into Id KO blastocysts. In the resultant embryos, LacZ-positive cells were detected in heart and forebrain (Fig.1C). At E11.5, all cardiac structures in rescued embryos were identical to those of WTs. No endocardial/myocardial/EC defects were observed (Fig.1C,F,I,L,O and not shown) and cell proliferation was restored (\%BrdU in compact myocardium:34 $\pm 4 \%$, see Fig. 1 Linset). $25 \%$ of the litter of Id1-/-Id3+/- (or Id1-/-Id2+/-) or $43.75 \%$ of the litter of Id $1-/-$ Id2+/-Id3+/- intercrosses should carry a null mutation in at least two Id genes, but double KOs never survive past E13.5 (Table SI). However, 15/75 (20\%) animals of the litter of Id1-/-Id3+/- and Id1-/-Id2+/-Id3+/- intercrosses with ES cell injection into blastocysts carried the genotype Id1-/-Id3-/-:R26; Id1-/-Id2-/-:R26; Id1-/-Id2+/-Id3-/-:R26 or Id1 $-/-\mathrm{Id} 2-/-\mathrm{Id} 3+/-$ :R26 past E13.5 (1/6 at E14.5, 5/25 at E17.5 and $9 / 45$ after birth). The appearance of double $\mathrm{KO}$ animals is therefore due to the presence of ES cells ( $\mathrm{p}$-value $<0.0001$ ).

Rescued hearts were structurally and functionally indistinguishable from those of WTs by histology and echocardiography (Fig.1P,R,S,V,W, $\mathrm{Z}_{1}, \mathrm{Q}, \mathrm{T}, \mathrm{U}, \mathrm{X}, \mathrm{Y}, \mathrm{Z}_{2}$ and table SII). Chamber walls/septum/OT/valves were normal (Fig.1P,R,S,V,W,Z $, \mathrm{Q}, \mathrm{T}, \mathrm{U}, \mathrm{X}, \mathrm{Y}, \mathrm{Z}_{2}$ and not shown). Unlike Id1/Id $3 \mathrm{KO}$ embryos, a continuous lining of endocardial cells apposed the myocardium in rescued pups, despite most endocardial cells not being ES cell-derived $\left(F i g 1 Z_{1}, Z_{2}\right)$. In rescued pups, body and heart sizes were severely reduced during the first three weeks, in some cases by $50-70 \%$ (P15 WT: $8.33 \mathrm{~g}$ and $65 \mathrm{mg}$ of body and heart weights respectively; P15 Id1 $-/-\mathrm{Id} 3-/-: \mathrm{R} 26: 3.11 \mathrm{~g}$ (63\% reduction) and $25 \mathrm{mg}$ ( $62 \%$ reduction) of body and heart weights respectively) and $44 \%$ of the rescued pups died during the first 4 weeks. The surviving rescued adult animals (5/9) displayed correction of these parameters (10-30\% reduction relative to WTs by $5-6$ weeks)(P120 Fig.1X,Y). The heart/body weight ratio in WT or rescued animals was constant [heart/body ratio:(7.9 \pm 0.5$) \times 10^{-3}$ ] and all organs were small and grosssly normal (not shown).

The average fraction of WT lacZ+ cells per heart was 20\% (range of incorporation:5-45\%) and blue cells were grouped in clusters (Fig.1Q,U,W,Y.). \%ES cell incorporation (in X-gal staining of tail sections) was confirmed by Southern analysis from adjacent tissue (Fig.S3A). An E14.5 Id1-/-Id3-/-:R26 rescued embryo with $15 \pm 5 \%$ of X-gal-positive cells displayed a 
WT band $80 \%$ less intense than the mutant band (Southern, Fig.S3A). To rule out that X-galnegative cells are ES-derived cells which had lost their ability to express LacZ, individual Xgal-negative cells were laser-captured from heart sections of an E17.5 Id1-/-Id2-/-:R26 rescued embryo and shown to be Id2-I- (Fig.S3B). R26 E17.5 heterozygous embryos stained uniformly blue after $\mathrm{X}$-gal staining indicating the result was not due to incomplete staining (Fig.1 Pinset).

In rescued hearts, a small subset of $\mathrm{R} 26$ cells from epicardium/endocardium/EC was the source of Id1/Id3 expression (Fig.S1H-J,M and not shown). R26 cells also populated the myocardium (Fig.S1K,L), but no Ids were detected (Fig.S1M and not shown). While it is likely that the rescue by ES cells is Id-dependent, it is formally possible that ES cells incorporated into the heart provide Id-independent signals. To test this, ES cells with reduced Id1 levels by siRNA knockdown (Fig.S3C) were injected into Id KO blastocysts. Id1 KD ES cells incorporated in the embryos (Fig.S3D), but failed to rescue Id1-/-Id3-/- blastocysts (Fig.S1E).

\section{Gene expression profiles in Id KO hearts are altered}

Microarray analysis of E11.5 WT and Id1/Id3 double KO hearts (table SIII) showed skeletal myosin alkali light chain (5) (skMLC) up-regulated 2.83-fold in mutant hearts. The enhanced signal was confirmed by Northern analysis ( +2.9 fold) (Fig.2E inset) and ISH (Fig.2E,F and not shown). Cardiac $\alpha$-myosin heavy chain ( $\alpha$-MHC), the adult isoform, was up-regulated (+2.0 fold) (table SIII). No other sarcomeric isoform was mis-regulated in Id-defective hearts (table SIII). A switch to a complete skeletal muscle program does not occur, since the myotomespecific bHLH factors MyoD and myogenin were not detected in the Id KO myocardium, and $\alpha$-cardiac actin was normally expressed (not shown). Stra13, a member of the Hey family of bHLH proteins ( 7) was up-regulated 4.92 fold in Id KO hearts (Table SIII), with enhanced Stra13 expression in the myocardium (Fig.2A,B). Stra13 was also detected in the neuroepithelium of the forebrain ganglionic eminence, and its expression was enhanced in the hemorrhagic Id1/Id3 KO embryos (Fig.2I,J). skMLC contains E-box motifs in its enhancer (8). Stra13 contains E-box motifs in the promoter and is regulated by Sharp-1, another bHLH member (9). $\alpha$-MHC is regulated by the bHLH dHand. Id loss may lead to ectopic activation of bHLH proteins that in turn activate Stra13, skMLC and $\alpha$-MHC.

\section{ES cells correct gene expression profiles in a non-cell autonomous manner}

$82 \%(60 / 74)$ of the mis-regulated markers, including skMLC, $\alpha$-MHC and Stra13 were corrected in chimeric rescued hearts (table SIV and Fig.2C,G). The myocardium not only displayed normal skMLC or Stra13 transcript levels in R26 cells but also in Id KO cells (Fig. $2 \mathrm{C}, \mathrm{D}, \mathrm{G}, \mathrm{H})$. Thus, signals emanating from the R26 cells (EC/endocardium/epicardium/ myocardium or distally outside the heart) revert the myocardium in a non-cell autonomous manner.

Most of the brain endothelium was composed of Id KO cells (Fig.2L). However, no collapse of the endothelium and associated hemorrhage was observed in rescued embryos (Fig.2K,L). Stra13 was also corrected in the neuroepithelium (Fig.2K). Although we cannot rule out that very few R26 endothelial cells account for the rescue, a non-cell autonomous mechanism operates since the whole of the brain endothelium appears normal.

\section{Supernatants of epicardial cultures containing R26-derived cells rescue proliferation defects of the Id KO hearts}

To determine if secreted factors from Id KO:R26 epicardium correct proliferation defects in the Id KO myocardium, we derived epicardial primary cells from WT, Id1-/-Id3+/- and Id1 
-/-Id3+/-:R26 adult hearts (10)(RT-PCR, Fig.3L) and collected conditioned medium (epiCM). WT and Id1-/-Id3+/-: R26 but not Id1-/-Id3+/- epicardial cells expressed Id1 (not shown). Id1-/-Id3+/- E17.5 whole hearts were cultured for 48 hours with epiCM and BrdU, sectioned and analyzed. WT and Id1-/-Id3+/-:R26 but not Id1-/-Id3+/- epiCM improved the proliferation rate of the Id KO compact myocardium relative to controls without epiCM (Fig.3E-H for Ki67 and 3E-Ginsets for BrdU). Since Id1-/-Id3+/- epiCM has little effect relative to controls with no epiCM (Fig3H), the R26 component of the KO:R26 epiCM (Fig3 Linset) accounts for the proliferation rescue. These results support the existence of an epicardial-to-myocardial rescue, through secretion of extracellular factors. We asked if misregulated myocardial markers could also be reversed. However, epiCM from WT or Id1-/-Id3 $+/-:$ 26 failed to correct skMLC in E12.5 Id1/Id3 KO hearts (Fig.3I-K).

\section{Injection of ES cells into females prior to conception partially corrects cardiac defects and rescues embryonic lethality of the Id KO offspring}

To investigate if myocardial proliferation is rescued by secretion of long-range ES celldependent factors, 3 month-old Id $1-/-I d 3+/-$ females were injected with $10^{5}$ R26 cells intraperitoneally (IP), and a week after mated with Id1 $1-/-\operatorname{Id} 3+/-$ males. Floating cells isolated from the IP cavity and cultured formed ES cell outgrowths (Fig.S3F) and embryoid bodies (not shown). R26 cells also adhered to connective tissue in the external side of the uterine wall, but no X-gal positive cells were detected inside the uterus, or in placenta or any part of the embryo including the yolk sac (not shown). $18 \%$ of newborn pups (7/40) carried the Id1-/-Id3-/genotype whereas no double knockout pups have been observed in over 10 years of breeding (p-value $<0.001$ ). Double KO pups contained no X-gal-positive cells (Fig.4G and not shown) and tail DNAs were LacZ-negative (PCR). Injection of supernatants from R26 cultures also rescued the lethality of Id1/Id 3 embryos (3/35), although at lower rates (9\%). The Id1/Id3 double KO newborn pups however, died by P2 and displayed enlarged hearts (body weight of Id1-/-Id3-/- or WT P1 pups: $1.4 \pm 0.1 \mathrm{~g}$; heart weight of Id1-/-Id3-/- P1 pups: $16 \pm 1 \mathrm{mg}$; heart weight of WT P1 pups: $10 \pm 1 \mathrm{mg}$; Id1-/-Id3-/- P1 heart/body ratio: $11.4 \pm 1.1 \times 10^{-3}$ compared to WT heart/body ratio: $7.1 \pm 0.6 \times 10^{-3} ; \mathrm{Id} 1-/-\mathrm{Id} 3+/-$ : R26 heart/body ratio at $\mathrm{P} 1$ was $7.3 \pm 0.5 \times 10^{-3}$ ) with muscular VSDs (Fig.4G), little endocardium and disorganized endothelium (Fig.4H). As with the Id KO hearts (with no injection), E11.5 rescued hearts (IP injection) displayed incomplete VS and discontinuous endocardium (Fig.4A, B). However, the thickness of the compact myocardium was corrected (Fig.4C; compare to Fig.1J-L) with increased myocardial proliferation (35 $\pm 4 \%$ BrdU incorporation at E11.5, Fig.4F,I insets for E18.5). P1 Id1/Id3 double KO hearts, no longer influenced by the R26 cells after birth, showed severe proliferation defects in the inner myocardium (Fig.4F,I).

$64 \%$ (47/74) of the markers mis-regulated in Id1/Id3 KO hearts were corrected at E11.5 after ES cell IP injection but $36 \%$ (27/74) failed to be restored (table SIV). skMLC was not corrected (table SIV). Mating of the same Id1-/-Id3+/- parents without additional R26 injections rendered no double KO pups. However, a subsequent mating with a second R26 injection led to a rescue, suggesting a reversible effect. WT murine embryonic fibroblasts (MEFs) were injected every three days in pregnant females, but no double KO pups were born. Although the brain vasculature of the Id double KO embryos IP rescued formed an endothelial cell aggregate at E11.5 (Fig.4B inset), no macroscopic hemorrhage was observed.

In support of the mode of action of ES cells at a distance, Id1-/-Id3-/-:R26 female chimeras were fertile, and when crossed with Id1-/-Id3+/- males, $44 \%$ of the litter (4/9) carried the Id1 $-/-\mathrm{Id} 3-/-$ genotype (PCR), although these neonates died soon after birth. Thus, ES-derived cells in chimeric mothers provide distal signals during pregnancy that rescue embryonic lethality. 


\section{Identification of IGF1 as the ES cell-secreted factor that partially corrects cardiac defects and rescues embryonic lethality of the Id KO offspring}

To identify ES cell-secreted factors responsible for the partial cardiac rescue, we performed microarray analyses from WT, KO or KO:R26 epicardial cultures. Because Id KO cultures (unlike WT or KO:R26 cultures) failed to rescue myocardial proliferation defects, we searched for secreted factors in WT or chimeric cultures with proliferation promoting capacity, whose expression was down-regulated in Id KO cultures. We identified several secreted factors that showed Id-dependent regulation (table SV [WT vs KO]: IGF1:+2, BMP4:-4.29, endothelin I:-3.25; table SVI [chimera vs KO]: IGF1:+1.62, BMP4:-1.52, endothelin-I:-1.62 fold). Of particular interest was IGF1 because it is down-regulated in Id KO cultures, it promotes cardiomyocyte proliferation (11), it is released in the bloodstream (12), Id1/Id3 and IGF1 KO embryos are small, and Id rescued chimeras as well as IGF1 KO pups are small with a variable rate of post-natal lethality $(13,14)$. IGF1 was also down-regulated in E11.5 Id1-/-Id3-/hearts $(-1.3$ fold, microarrays).

As in the case of Ids, IGF1 was observed at E11.5 in non-myocardial layers of developing hearts (EC/endocardium/epicardium), but not in the myocardium (Fig.5A,C,E). IGF1 expression was compromised in the endocardium/epicardium of Id1-/-Id3-/- E11.5 hearts. (Fig.5B,D,F). Since IGF1 expression is not fully abrogated by Id1/Id3 loss, it is possible that Id2 sustains basal levels of IGF1 expression. We also observed down-regulation of the $8-\mathrm{Kb}$ message of IGF1 in Id1-/-Id3-/- E11.5 livers (Fig.5G), the main source of circulating IGF1. IGF-binding protein-4 (IGFBP4), an inhibitor of IGF1 activity, was up-regulated in Id1/Id3 KO tissues (Fig.5G and Table SV [WT vs KO]:-1.85 fold and Table SVII [KO vs WT]:+4.59).

We hypothesized that IGF1 from ES cells (Fig.S3G) is released into maternal circulation, passes through the placenta and accounts for the partial rescue of Id1-/-Id3-/- embryos. We injected IGF1 (700 ng/day) intraperitoneally into Id1-/-Id3+/- pregnant females, resulting in an increase in serum levels of IGF1 (Fig.S3H). 6/41 (15\%) of newborn pups carried the Id1-/ - Id3 $-/$ - genotype (p-value $<0.001$ ). Thus, maternal injection of IGF1 recapitulates the effect observed by maternal injection of ES cells. To test specificity of the effect and because loss of Id1 down-regulates expression of parathyroid hormone (PTH) in ES cells (not shown) we injected recombinant PTH intraperitoneally, but no Id1/Id3 pups were born (0/15). Similar to IP injection of R26 cells, pups born from IGF1-injected mothers died by P2, the rescued hearts were hypertrophic, with muscular VSDs and compromised endocardium/endothelium but with normal myocardial wall (Fig 4J-L). Thus IGF1 is one of the Id-dependent non-cell autonomous factors responsible for the rescue of myocardial proliferation defects.

\section{WNT5a corrects cardiac gene expression profiles}

Although maternal injection of ES cells (or IGF1) rescues embryonic lethality and proliferation defects in Id1/Id3 KO hearts, Id1/Id3 KO neonates display cardiac abnormalities with altered gene expression profiles. Because the rescue in chimeric hearts generated by injection of WT ES cells in blastocysts is complete, we hypothesized that a local requirement for ES cells, which is missing in the IP rescue experiment, was critical for a full rescue. We therefore searched for locally-secreted factors up-regulated in the chimeric epicardial arrays relative to Id knockout controls. WNT5a, a non-canonical WNT is a lipid-modified glycoprotein associated with the cell membrane and extracellular matrix (15), and was up-regulated 2.3-fold in chimeric versus KO as well as WT epicardial cultures (Tables SV and SVI), suggesting a possible neomorphic effect of ES cells incorporated in the epicardium. Consistently, WNT5a was ectopically expressed in the epicardium of E16.5 chimeric rescued hearts (Fig.S4). To determine if WNT5a corrects gene expression profiles, we performed co-culture experiments in which heart explants (P1 Id1-/-Id3-/- hearts partially rescued by maternal IP injection of 
R26 cells [IP R26]) were grown on MEFs over-expressing WNT5a (3 fold up-regulation, microarrays). 653 genes were mis-regulated between WT and Id1/Id3 KO (IP R26) heart explants in the presence of mock-infected MEFs (table SVII), including components of the WNT pathway (table SVII [KO vs WT]: $\beta$-catenin:-2.64, $\beta$-catenin interacting protein: +2.3 , Dkk3:-2.14 fold). 554/653 genes (85\%) were corrected when Id1/Id3 KO (IP R26) heart explants were cultured on WNT5a over-expressing MEFs, including $\beta$-catenin, $\beta$-catenin interacting protein and Dkk3. The adult isoform of cardiac MHC $(-12.13$ fold down-regulated in the Id KO explants) was corrected in the presence of WNT5a-over-expressing MEFs (table SVII). It is likely that ES cells circumvent Id loss in embryonic hearts by providing WNT5a locally which can normalize gene expression profiles.

\section{Discussion}

In this report we demonstrate that compound Id1,3 knockout embryos have severe cardiac defects that result from a failure in signaling between the Id expressing epicardium and endocardium and the Id negative myocardium. This failure results in a thinning of the myocardial wall due to proliferation defects, ventricular septal defects, endocardial and endocardial cushion defects and outflow tract atresia. We have been able to show that cardiac defects can be rescued by incorporation of wild type ES cells into the heart with as little as $20 \%$ chimerism. Transcriptional alterations observed in myocardial cells of Id KO hearts are largely restored to normal in Id $\mathrm{KO}$ cells of the chimeric hearts indicating a non-cell autonomous rescue by wild type ES cells. As in the case of Id mutant embryos, other congenital heart malformations appear to partially result from non-cell autonomous defects in cell proliferation (16-18). Although injection (aggregation) experiments with KO ES cells and WT blastocysts (morulas) have been previously reported with non-cell autonomous correction of cardiac defects $(16,19,20)$, this is the first time that low numbers of WT ES cells have been injected into blastocysts fated to die with the resultant production of viable and fertile adult animals.

The rescue we show is due to a combination of long-range and short-range effects of the injected ES cells. IGF-1, secreted by ES cells, is lower in Id KO embryos relative to wild type and is restored by ES cells in chimeras or by intraperitoneal (IP) injection of ES cell into mothers bearing Id 1,3 compound mutant embryos prior to conception. This latter result suggests that IGF-1 is a long-range acting factor since ES cells do not cross placenta. The rescue however by IP injection of ES cells and direct injection of recombinant IGF-1 is incomplete with enhanced proliferation of the myocardium observed but many of the structural cardiac defects still remaining, including ventricular septal and endocardial/endothelial defects. Thus, the VS/ endocardial/endothelial defects and myocardial proliferation defects are separable and even if VS/endocardial/endothelial defects contribute to a defect in myocardial proliferation this contribution can be overcome by IGF1 administration.

Reversion of transcriptional defects of Id KO myocardial cells and repair of the structural defects is not achieved with IGF-1or ES cell IP injection alone. Rather short-range effects of ES cells incorporated into chimeric hearts are required to see this full rescue of the Id mutant heart phenotype. We have identified WNT5a, a non-canonical member of the WNT signaling family, as likely playing a key role in the rescue since MEFs expressing WNT5a are capable of reverting myocardial markers from knockout to wild type levels in $85 \%$ of the loci examined in co-culture experiments. Interestingly, ES cells in chimeras are probably producing a neomorphic effect with respect to WNT5a expression since the chimeric rescued embryos have a broader WNT5a expression pattern than observed in wild type embryos. Whether this broadening of WNT5a expression is required for the near complete marker reversion observed in most of the Id KO cells in chimeric hearts has not been demonstrated. 
The ES cell rescue reported here is dependent on the expression of the Id genes since ES cells treated with Id 1 siRNA fail to effect the rescue. Interestingly, the Ids have been shown to be sufficient for self-renewal in ES cells in culture (21). While the levels at which Id is required in ES cells for the cardiac rescue observed have yet to be fully elucidated, maintenance of IGF-1 expression and expansion of the WNT5a expression pattern are likely to be critical components. In any event, it is clear from these studies ES cells can provide factors missing from mutant mammalian embryos and induce neomorphic effects that can compensate for the effects of the mutation. Such properties may imbue ES cells with a greater therapeutic value than previously imagined.

\section{References}

1. Ruzinova MB, Benezra R. Trends Cell Biol Aug 2003;13:410. [PubMed: 12888293]

2. Jen Y, Manova K, Benezra R. Dev Dyn Nov 1996;207:235. [PubMed: 8922523]

3. Jen Y, Manova K, Benezra R. Dev Dyn Jan 1997;208:92. [PubMed: 8989524]

4. Lyden D, et al. Nature Oct 14 1999;401:670. [PubMed: 10537105]

5. Kelly R, Alonso S, Tajbakhsh S, Cossu G, Buckingham M. J Cell Biol Apr 1995;129:383. [PubMed: 7721942]

6. Bouillet P, et al. Developmental Biology 1995;170:420. [PubMed: 7649373]

7. Boudjelal M, et al. Genes Dev Aug 15 1997;11:2052. [PubMed: 9284045]

8. Kelly RG, et al. Dev Biol Jul 15 1997;187:183. [PubMed: 9242416]

9. Azmi S, Sun H, Ozog A, Taneja R. J Biol Chem May 30 2003;278:20098. [PubMed: 12657651]

10. Chen TH, et al. Dev Biol Oct 1 2002;250:198. [PubMed: 12297106]

11. Reiss K, et al. Proc Natl Acad Sci U S A Aug 6 1996;93:8630. [PubMed: 8710922]

12. Adamo M, Lowe WL Jr, LeRoith D, Roberts CT Jr. Endocrinology Jun 1989;124:2737. [PubMed: 2721444]

13. Powell-Braxton L, et al. Genes Dev Dec 1993;7:2609. [PubMed: 8276243]

14. Liu JP, Baker J, Perkins AS, Robertson EJ, Efstratiadis A. Cell Oct 8 1993;75:59. [PubMed: 8402901]

15. Cadigan KM, Nusse R. Genes Dev Dec 15 1997;11:3286. [PubMed: 9407023]

16. Tran CM, Sucov HM. Development May 1998;125:1951. [PubMed: 9550727]

17. Hatcher CJ, et al. Dev Biol Feb 15 2001;230:177. [PubMed: 11161571]

18. Chen J, Kubalak SW, Chien KR. Development May 1998;125:1943. [PubMed: 9550726]

19. Wu H, Lee SH, Gao J, Liu X, Iruela-Arispe ML. Development Aug 1999;126:3597. [PubMed: 10409505]

20. Clouthier DE, Williams SC, Hammer RE, Richardson JA, Yanagisawa M. Dev Biol Sep 15 2003;261:506. [PubMed: 14499656]

21. Ying QL, Nichols J, Chambers I, Smith A. Cell Oct 31 2003;115:281. [PubMed: 14636556]

22. We thank J. Massague and members of the Benezra lab for critically reading the manuscript, W. Mark, J.-H. Dong (Transgenic Facility), A. Viale (Microarray Facility), S. Curelariu (technical assistance), P. Soriano (R26 cells), Y. Yokota (Id2 deficient mice), V. Mittal and S. Gupta (Id1 knock-down ES cells) and the Molecular Cytology Core Facility. D.F. and R.B are funded by NIH grants (KO1HL076568-01 and R01CA107429 respectively). 


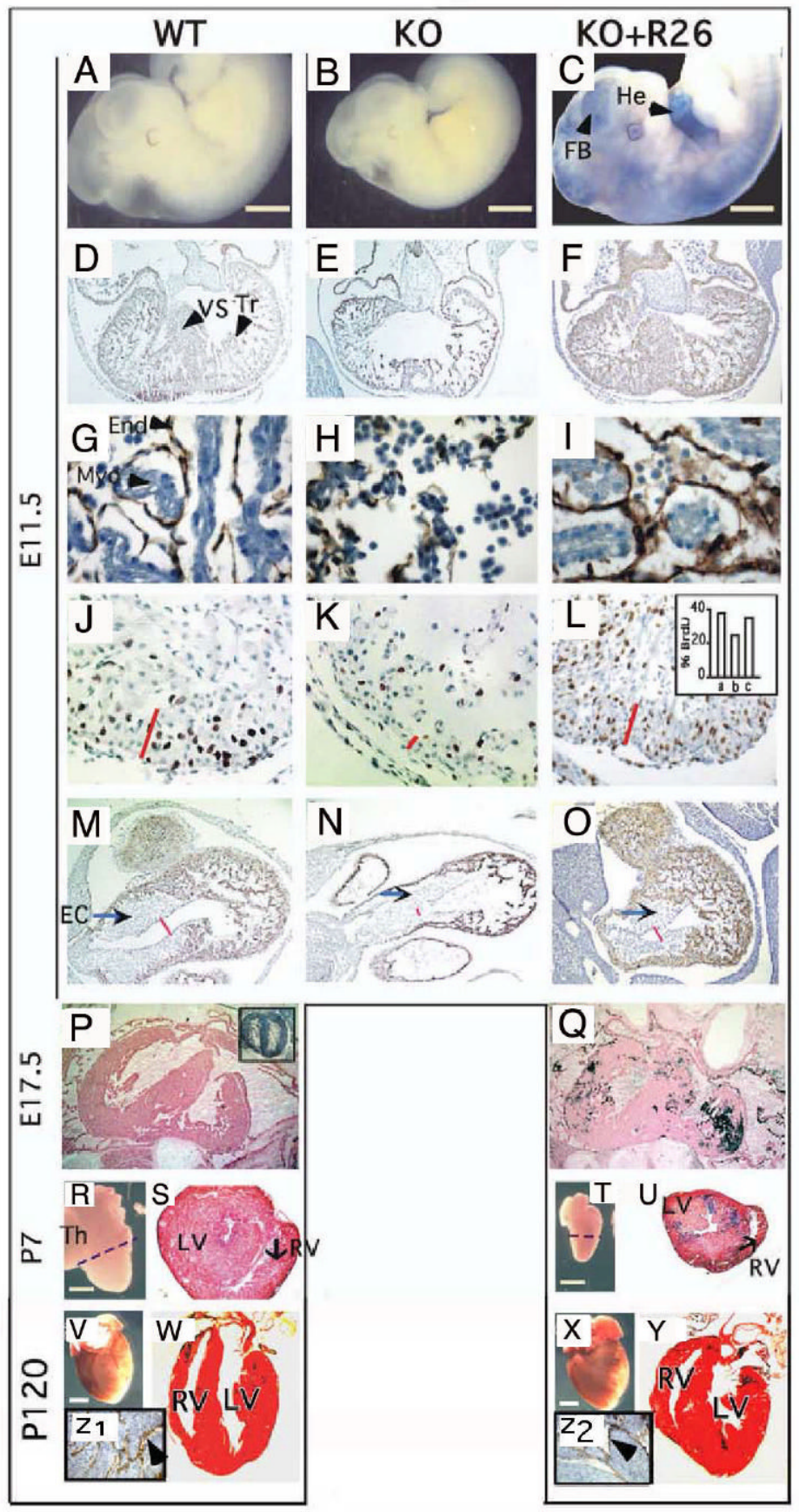

Figure 1.

Cardiac defects in Id KO embryos are rescued by injection of 15 ES cells. A-O, A WT (A,D,G,J,M), Id1-/-Id3-/- (B,E,H,K,N) or Id1-/-Id3-/-:R26 (C,F,I,L,O) E11.5 embryo was X-gal stained (A-C) or transversely sectioned at ventricle (D-L) or OT level (M-O). DF, (50X) and M-O, (80X), desmin immunodetection. G-I, CD31 immunodetection (200X). J-L, BrdU immunodetection (100X). L inset, \%BrdU from J-L. P-Q, A WT (P), ROSA+/(Pinset) or Id1-/-Id3-/-:R26 (Q) E17.5 embryo was X-gal/eosin stained (50X).

$\mathbf{R}, \mathbf{S}, \mathbf{V}, \mathbf{W}, \mathbf{Z 1}, \mathbf{T}, \mathbf{U}, \mathbf{X}, \mathbf{Y}, \mathbf{Z 2}$, A WT (R,S), WT:R26 (V,W), Id1-/-Id2+/-Id3-l-:R26 (T,U) or Id1-/-Id3-/-:R26 (X,Y) P7 (R-U) or P120 (V-Y,Z1,Z2) heart (R,T,V,X), was sectioned (dashed lines), X-gal/eosin stained (S,U,25X, W,Y,15X) or CD31 immunostained 
(Z1,Z2,50X). He:heart; FB:forebrain; VS:ventricular septum; Tr:trabeculae; EC:endocardial cushion; End:endocardium; Myo:myocardium; Th:thymus; LV:left ventricle; RV:right ventricle; blue arrow:outflow tract EC and red bar:luminal thickness (M-O); red bar:myocardial wall thickness (J-L); black arrowhead:CD31+ cells $(\mathbf{Z 1 , Z 2 )}$. Scale bar $500 \mu \mathrm{m}(\mathbf{A}-\mathbf{C})$ or $2 \mathrm{~mm}(\mathbf{R}, \mathbf{T}, \mathbf{V}, \mathbf{X})$. KO: Id KO. 

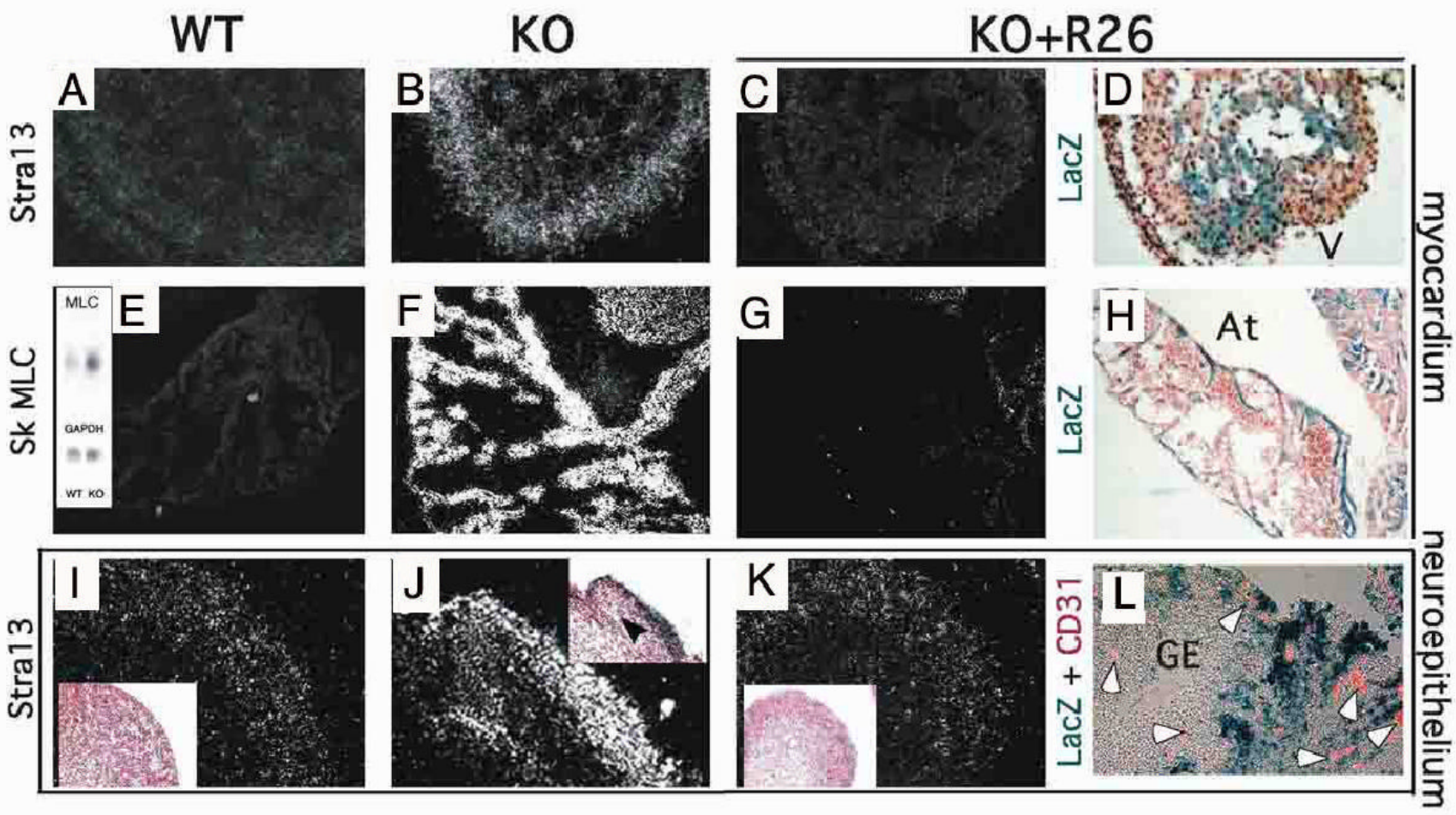

Figure 2.

R26 cells rescue Id1-/-Id3-/- cells non-cell autonomously. A-C, I-K, A WT (A,I), Id1-/-Id3 $-/-(\mathbf{B}, \mathbf{J})$ or Id1-l-Id3-/-:R26 (C,K) E11.5 embryo was subjected to ISH for Stra13 (100X). $\mathbf{D}$, adjacent section of $\mathbf{C}$, X-gal stained. L, A Id1-/-Id3-l-:R26 E14.5 embryo was X-gal- and CD31 immuno-stained (100X). E-G, A WT (E), Id1-/-Id3+/-(F) or Id1-/-Id2+/-Id3-/-:R26 (G) P7 heart was subjected to ISH for skMLC(50X). $\mathbf{H}$, adjacent section of $\mathbf{G}, \mathrm{X}$-gal stained. E (inset), northern blot from a WT or Id 1-/-Id3+/-E13.5 heart probed for skMLC. I-K (insets), bright field; V:ventricle ; At:atrium; GE:ganglionic eminence; black arrowhead: hemorrhage (J,inset); white arrowheads: CD31+ cells $(\mathbf{L})$. 


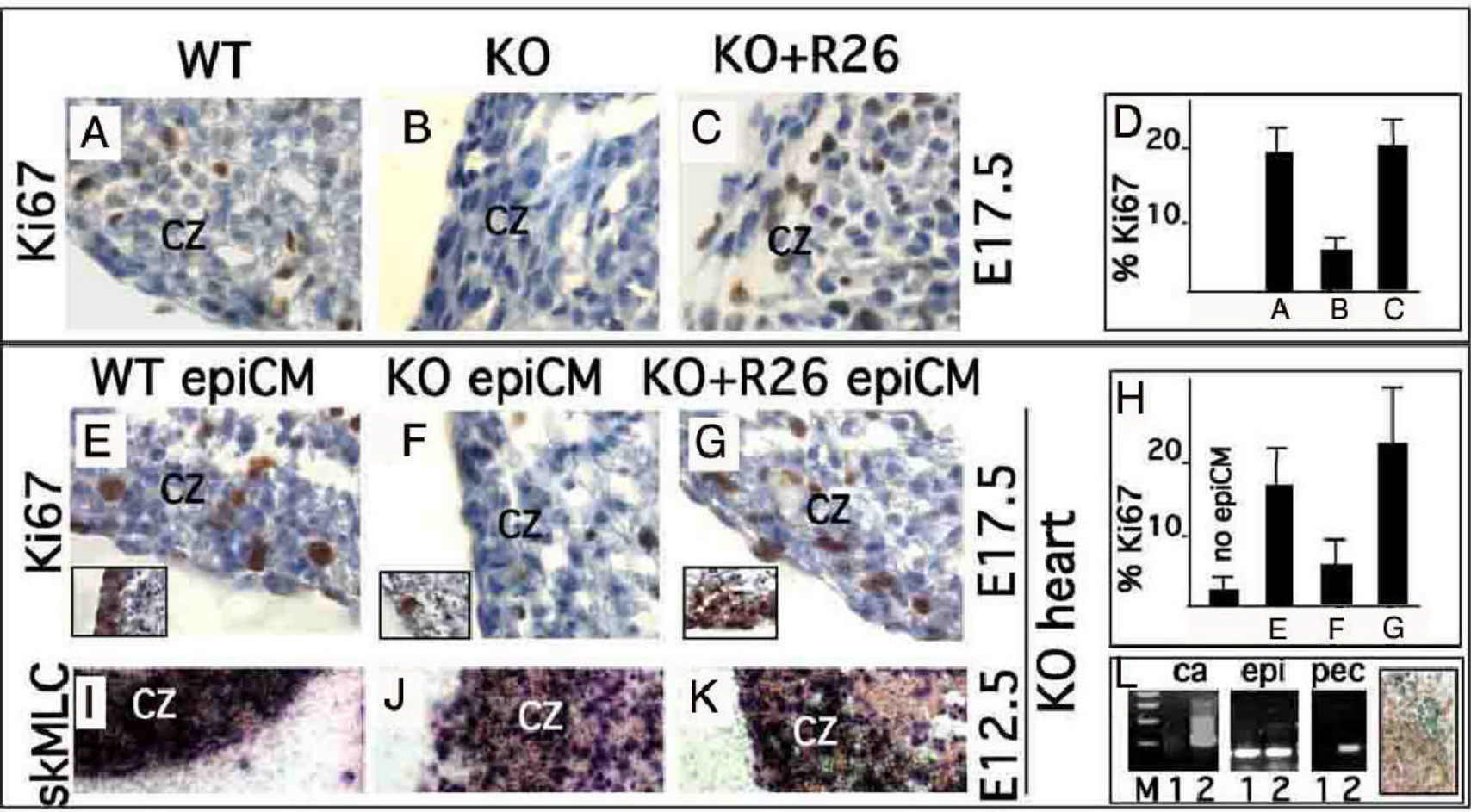

Figure 3.

Conditioned medium from Id KO:R26 epicardial cells (epiCM) corrects myocardial proliferation defects in Id KO hearts. A-C, A WT, Id1-/-Id3+/- or Id1-/-Id3+/-:R26 E17.5 embryo was sectioned at heart level and Ki67 immunostained. D, \% Ki67+ cells of A-C. EG and I-K, An Id1-/-Id3+/-E17.5 (E-G) or Id1-/-Id3-/-E12.5 (I-K) heart was cultured with BrdU and WT (E,I), Id1-/-Id3+/- (F,J) or Id1-/-Id3+/-:R26 (G,K) epiCM, sectioned and Ki67 (E-G) or BrdU (E-Ginsets) immunostained or subjected to ISH for skMLC (I-K). H, \% Ki67+ cells of E-G and control with no epiCM. L, RT-PCR (cardiac actin, epicardin, pecam) of Id1-/-Id3+/-:R26 epicardial-derived cells (lanes1) or E13.5 heart (lanes2). L inset, Id1-/ -Id3+/-:R26 epicardial derived-cells were X-gal stained. KO:Id1-/-Id3+/-; R26:Rosa26. Magnification:630X (A-C and E-G); 400X (I-K); CZ:myocardial compact zone. M:markers. 

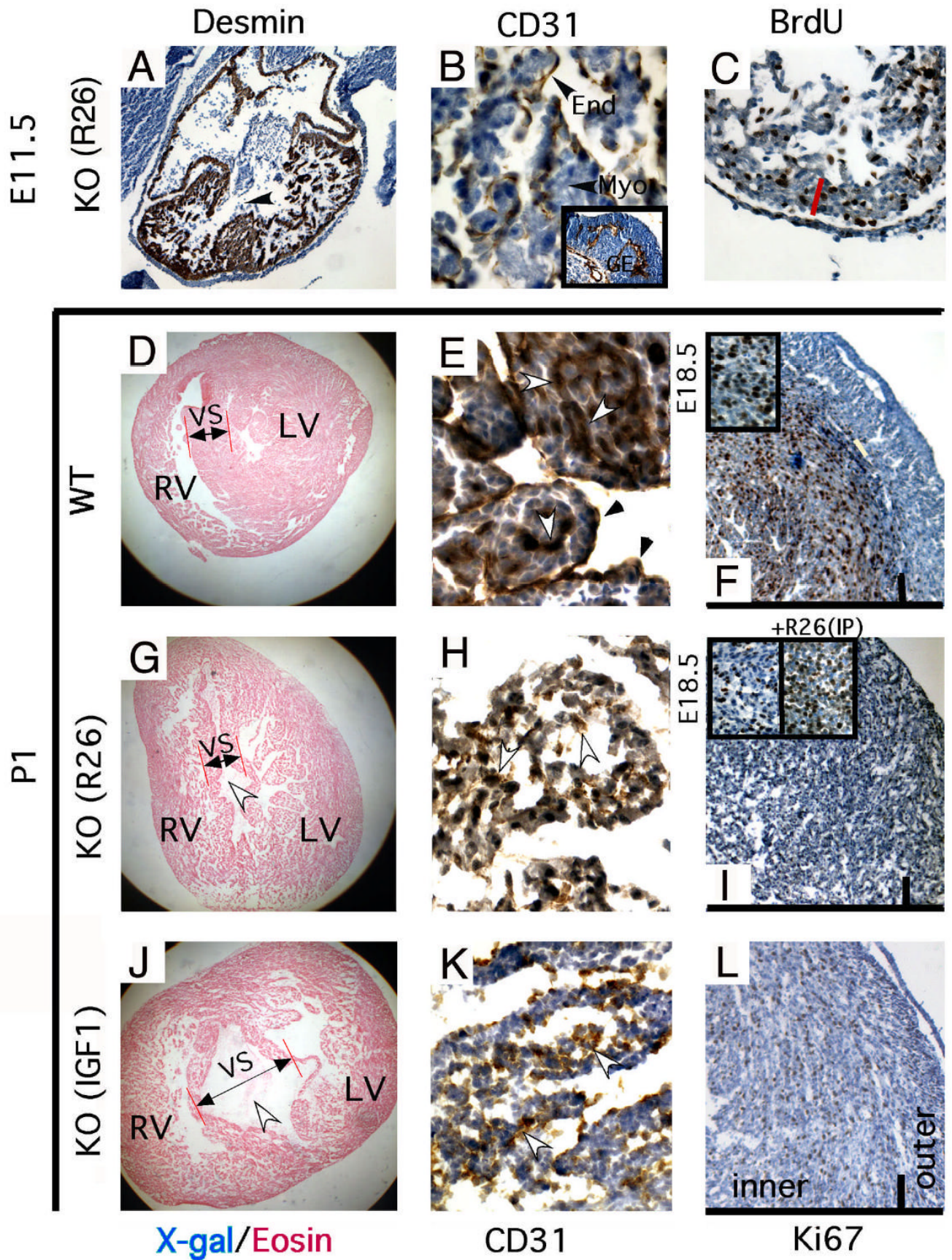

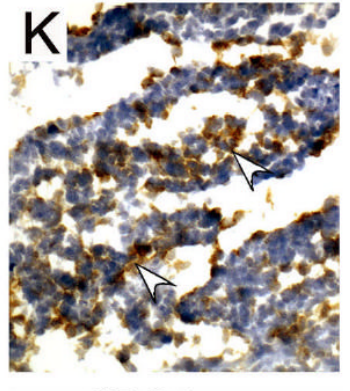

CD31
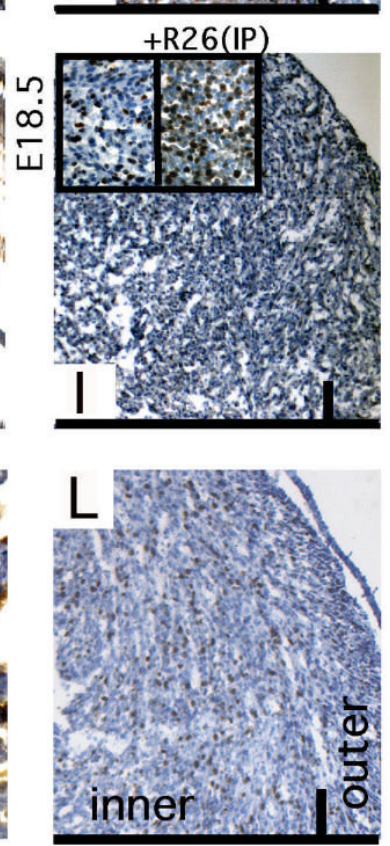

Ki67

Figure 4.

Id1/Id3 double KO pups are born from mice intraperitoneally injected with ES cells or IGF1. A-C, An Id1-/-Id3-/- E11.5 embryo from an ES-injected female was sectioned at heart (AC) or brain (Binset) level and Desmin (A,50X), CD31 (B,200X and B inset, 100X) or BrdU (C,100X) immunostained. D-L, A WT (D-F), an Id1-/-Id3-/- from an ES-injected female (G-I) or from IGF1-injected female (J-L) P1 heart was transversely sectioned and X-gal/eosin (D,G,J,25X), CD31 (E,H,K,200X) or Ki67 (F,I,L,50X) stained. F,I (insets), A WT (F,inset, $400 \mathrm{X}$ ) or an Id1-/-Id3+/- E18.5 heart from a non- or from an R26 injected female (Iinsets, 400X) was sectioned and Ki67 immunostained. KO(R26): Id KO embryos (pups) from R26 injected females. $\mathrm{KO}(\mathrm{IGF} 1)$ : Id KO pups from IGF1 injected females. End:endocardium; 
Myo:myocardium; RV:right ventricle; LV:left ventricle; VS:ventricular septum; GE:ganglionic eminence; arrowhead (A):VS defect; red bar (C):thickness of compact myocardium; white arrowhead (G):VS defect; black arrowheads $(\mathbf{E})$ :CD31+endocardial cells; white arrowheads $(\mathbf{E})$ :CD31+ endothelial cells; white arrowheads $(\mathbf{H}, \mathbf{K})$ :disorganized endothelial cells. 

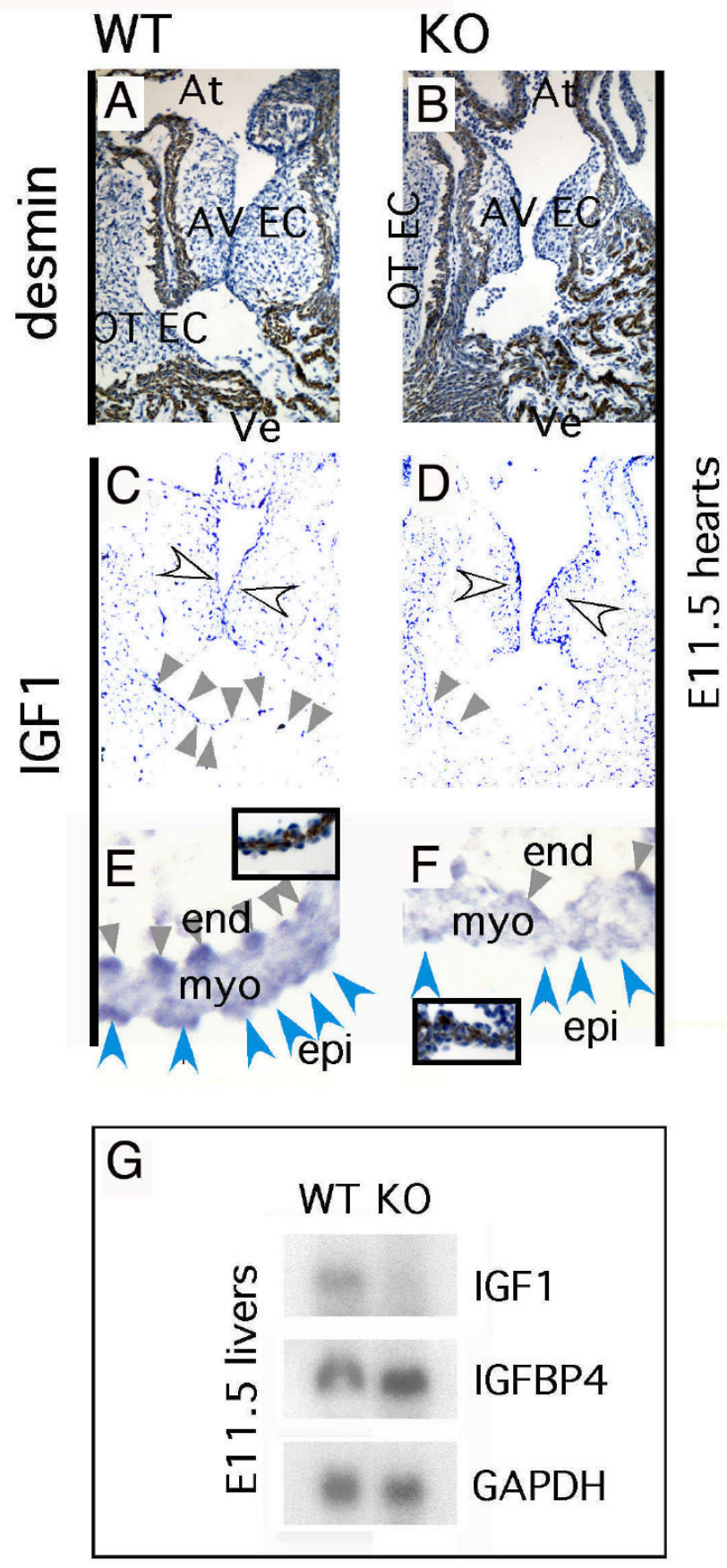

Figure 5.

IGF1 is down-regulated in Id1/Id3 KO embryos. A-F, A WT (A,C,E) or an Id1-/-Id3-/(B,D,F) E11.5 heart was sagittally sectioned and desmin immuno- $(\mathbf{A}, \mathbf{B})$ or IGF1 in-situ- $(\mathbf{C}-$ F) stained. E,F (insets), desmin immunostaining of E,F. A-D (100X); E,F (200X). G, RNA from a WT or an Id1-/- Id3-/- E11.5 liver was hybridized with IGF1, IGFBP4 or GAPDH. At:atrium; OT EC:outflow tract endocardial cushion; AV EC:atrio-ventricular EC, Ve;ventricle; end:endocardium; epi:epicardium. White arrowheads (C,D):EC+ cells; grey arrowheads $(\mathbf{C , D})$ :endocardial+ cells; grey arrowheads $(\mathbf{E}, \mathbf{F})$ :end+ and epi+ cells. 